\section{Orale Provokationen beeinflussen die Lebensqualität in allen Altersgruppen}

Menschen essen, um das Gefühl von Sättigung zu verspüren. Im günstigsten Fall werden durch die Nahrungsaufnahme alle lebenswichtigen Makro- und Mikronährstoffe zugeführt. Darüber hinaus ist Essen aber auch ein wichtiger Bestandteil vieler sozialer Funktionen, sodass eine Nahrungsmittelallergie deutliche Einschränkungen der Lebensqualität nach sich zieht. Eine aktuelle Studie hat den Einfluss von oralen Provokationen auf die Lebensqualität untersucht.

E Itern betroffener Kleinkinder empfinden eine Zunahme an Lebensqualität nach einer doppelblinden, placebokontrollierten Provokation (DBPCFC) und zwar unabhängig vom Ausgang der Provokation. Diese Erkenntnis wurde nun durch eine niederländische Arbeitsgruppe untermauert und erweitert: van der Velde und Mitarbeiter zeigten eindrucksvoll, dass Veränderungen auf die Lebensqualität nach DBPCFC auch bei Erwachsenen, Jugendlichen und Kindern nachzuweisen sind. Die Autoren ließen 221 Patienten unterschiedlicher Altersgruppen ([n = 73] $\geq 18$ Jahre, [n = 71]: 13-17 Jahre bzw. [n = 77]: 8-12 Jahre), bei denen eine DBPCFC geplant war, zu zwei Zeitpunkten Fragebögen ausfüllen. Gefragt wurde nach der krankheitsbezogenen Lebensqualität bzw. der Schwere der Nahrungsmittelallergie und dem damit verbundenen Risiko. Die Interventionsgruppe ( $\mathrm{n}=53$ bzw. 46 bzw. 57) füllte die Fragebögen einen Monat vor sowie sechs Monate nach Provokation aus, die Kontrollgruppe ( $\mathrm{n}=20$ bzw. 25 bzw. 20) füllte die gleichen Fragebögen im Sieben-Monate-Intervall aus, ohne dass eine Provokation in dieser Zeit durchgeführt wurde.

Die Lebensqualität verbesserte sich in allen Altersgruppen deutlich, wenn die Provokationen ohne Reaktionen erfolgten ( $n=24$ bzw. 13 bzw. 23). Bei Kindern führte - ähnlich wie bei Eltern von betroffenen Kleinkindern - selbst die Bestätigung der Allergie zu einer Verbesserung der Lebensqualität. Die Angabe einer stark geminderten Lebensqualität zu Beginn der Untersuchung wirkte sich begünstigend auf die Lebensqualität nach sieben Monaten aus. Dagegen ging die klinische Relevanz von mehr als zwei Nahrungsmittelallergenen im Kindesalter mit einer Verschlechterung der Lebensqualität nach DBPCFC einher.
Fazit: Die Untersuchung unterstreicht, welche Bedeutung Nahrungsmittelprovokationen nicht nur im Rahmen der Diagnostik, sondern auch langfristig für die Lebensqualität der Betroffenen haben. Vor allem ein negatives Ergebnis erhöht die Lebensqualität deutlich. Beeindruckend ist der hohe Anteil der negativen Provokationen in allen Altersgruppen: Bei knapp der Hälfte der Erwachsenen bzw. Kindern und bei einem Drittel der Jugendlichen verlief die DBPCFC ohne Reaktion, d.h. mindestens eine der vorliegenden Sensibilisierungen war ohne klinische Relevanz. Bei sechs von 24 Erwachsenen, vier von 13 Jugendlichen und vier von 23 Kindern konnte eine Nahrungsmittelallergie durch die Provokationen komplett ausgeschlossen werden.

van der Velde JL et al. Food allergy-related quality of life after double-blind, placebocontrolled food challenges in adults, adolescents, and children. J Allergy Clin Immunol 2012; 130: 1136-43

Kommentar: Im Kindesalter hat selbst ein positives Provokationsergebnis eine beträchtliche Steigerung der Lebensqualität zur Folge. Diese Beobachtung macht deutlich, wie stark das Gefühl der Sicherheit, zumindest in dieser Altersgruppe, die Lebensqualität positiv beeinflussen kann. Das Wissen, dass die vorliegende Sensibilisierung klinisch relevant ist, macht es den Betroffenen offenbar leichter, den täglichen Umgang mit der Nahrungsmittelallergie zu meistern, und ebnet möglicherweise den Weg, eine professionelle Ernährungstherapie in Anspruch zu nehmen.

Im Jugendalter hat ein positives Provokationsergebnis hingegen keine Verbesserung der Lebensqualitätzur Folge.Möglicherweise ist die Bestätigung der Nahrungsmittelallergie in diesem Alter schwieriger zu verkraften, weil die Konsequenzen vor allem in Bezug auf die „peer-group" schwerer wiegen als die
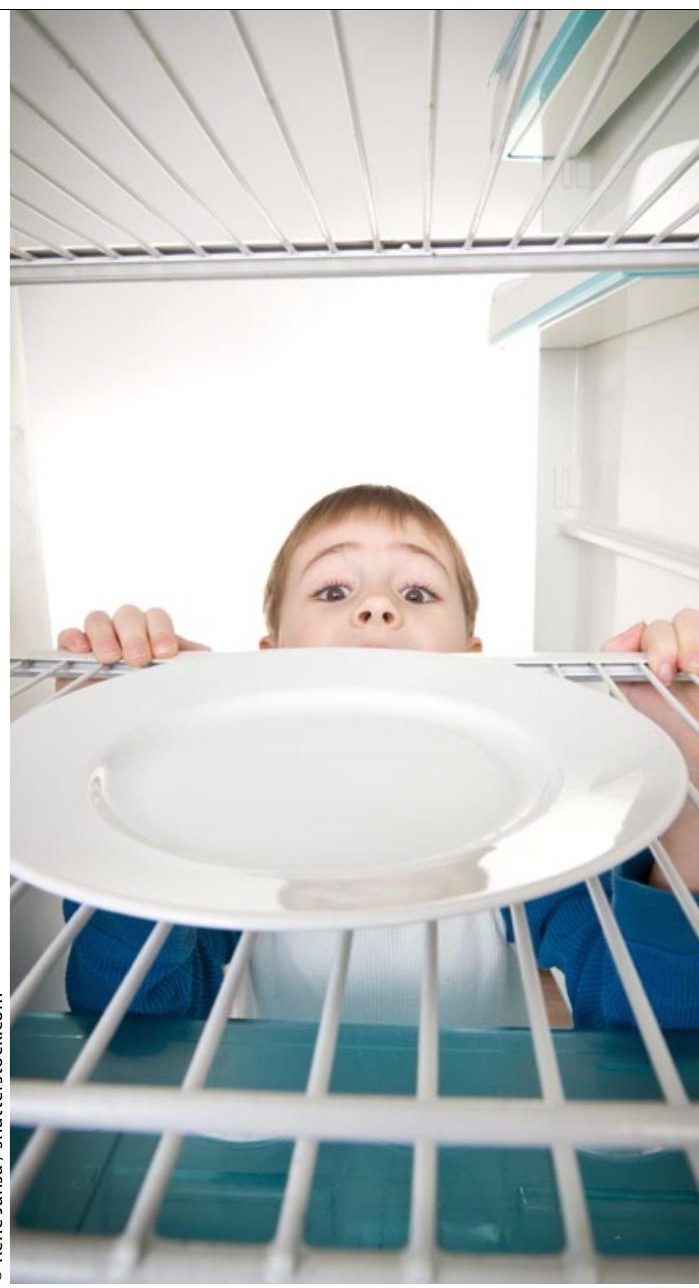

Schon zu wissen, was auf den Teller kommen darf, kann die Lebensqualität erhöhen - zumindest bei Kindern und deren Eltern.

gewonnene Sicherheit. Zusätzlich könnte die zeitnahe Erfahrung allergischer Reaktionen den Lösungsprozess von den Eltern erschweren und einen Hinderungsgrund darstellen, die eigene Nahrungsmittelallergie zu verdrängen. Auch die Konsequenz, eine professionelle Ernährungstherapie in Anspruch zu nehmen, könnte in dieser Altersgruppe eher Unwillen erzeugen und damit negativ bewertet werden. Die Beobachtung einer ausbleibenden Verbesserung der Lebensqualität im Jugendalter sollte allerdings nicht dazu führen, auf orale Provokationen in dieser Altersgruppe zu verzichten. Vielmehr sollten Jugendliche nach einem positiven Provokationsergebnis aufgefangen und in ihren Ängsten ernst genommen werden. Dabei kommt der Unterstützung von Seiten des Therapeuten (Arzt, Ernährungsfachkraft) in diesem Alter eine besondere Bedeutung zu.

Dr. Imke Reese, München 